\title{
Dynamic TEM Observation of Gold Nanoparticles during Catalytic Reactions at Ambient Pressure
}

\author{
T. Kawasaki****, T. Miura*, Z. Cui* and T. Tanji*****
}

* Dept. Electrical Eng., Nagoya Univ., Furo-cho, Chikusa-ku, Nagoya, 464-8603, Japan

** EcoTopia Science Inst., Nagoya Univ., Furo-cho, Chikusa-ku, Nagoya, 464-8603, Japan

*** Global Research Center for Environment and Energy based on Nanomaterials Science (GREEN), 1-2-1 Sengen, Tsukuba, 305-0047, Japan

It wad discovered that gold exhibits catalytic activity when it is in the form of fine particles having a size of less than $10 \mathrm{~nm}$ and is tightly supported on specific metal oxides [1]. In order to understand its catalytic behavior, in-situ observation under ambient reaction condition by environmental transmission electron microscope (ETEM) is quite essential [2-3]. The authors had revealed that the shape of catalytic gold nanoparticles is deformed during $\mathrm{CO}$ oxidation, even at relatively low gas pressure $(500-1000 \mathrm{~Pa})$ [3-4]. In the paper, we report about in-situ observation of gold catalysts at up to ambient atmospheric pressure.

In our windowed-type environmental-cell system [5], a co-axial-type gas-flow specimen holder [6] with C/SiN hybrid sealing-films [7] is inserted into a conventional $200 \mathrm{kV}$ TEM (H-8000; Hitachi). Dynamic TEM images were sequentially recorded using a CCD Camera (TVIPS; F114) for every 0.1 seconds with 12 -bit grayscale level at $512 \times 512$ pixels. A gold catalyst was prepared by the deposition precipitation method, mixed with rod-shaped $\mathrm{TiO}_{2}$ powder with an anatase structure as a support. The reaction gas consisted of $1 \% \mathrm{CO}$ in artificial dry air $\left[\mathrm{N}_{2}(78 \%)\right.$ $\left.+\mathrm{O}_{2}(21 \%)\right]$. The reaction may occur as $2 \mathrm{CO}+\mathrm{O}_{2}=2 \mathrm{CO}_{2}$ on the catalyst surface. The total pressure of the gas was set at $10^{3}-10^{5} \mathrm{~Pa}$ (atmospheric pressure).

Figure 1(a) - (h) shows a result of dynamic observation of a gold catalyst at $1 \times 10^{3} \mathrm{~Pa}$. They correspond to TEM images taken sequentially at intervals of 0.2 seconds. A gold nanoparticle is surrounded by white broke lines to emphasize its profile in each image. This result clearly show that the particle exhibited crystal habit and the surface mainly consists of (111), (100) and (110) facets. As an example, Fig. 1(i) corresponds to a schematic illustration of the Wulff construction of Fig. 1(h). In addition, the sequential observation revealed that the shape of the gold particle was changing during catalytic reaction. For quantitative analyses, tilt angle of the particle, and sizes of three facets and interface were measured in each images and were plotted in Fig. 2; the former was defined as an angle between the (100) facet and the substrate surface, and the latter was determined as total profile length of each facet and interface width (see Fig. 1(i)). The graph of Fig. 2 indicates that this particle wobbled randomly at the angle of $\pm \sim 10$ degrees, and the sizes of (111), (110) facets were largely varied, though the interface width was almost constant. This means that there is strong connection between the gold and the $\mathrm{TiO}_{2}$ substrate but shape of 
the particle was easily fluctuated due to the heat-of-reaction of $\mathrm{CO}$ oxidation. Additionally, such structural variations were also dependent on surrounding gas conditions.

\section{References}

[1] M. Haruta, Catalysis Today 36 (1997) 153.

[2] S. Giorgio et al., Ultramicroscopy 106 (2006) 503-507.

[3] K. Ueda et al., Surf. Interface Anal. 40 (2008) 1725.

[4] T. Kawasaki et al., Proc. M\&M2010. (2010) 1816.

[5] T. Kawasaki et al., Rev. Sci. Inst. 80 (2009) 113701.

[6] T. Kawasaki et al., Proc. M\&M2010. (2010) 332.

[7] T. Kawasaki et al., Proc. M\&M2011. (2011) in the present conference.
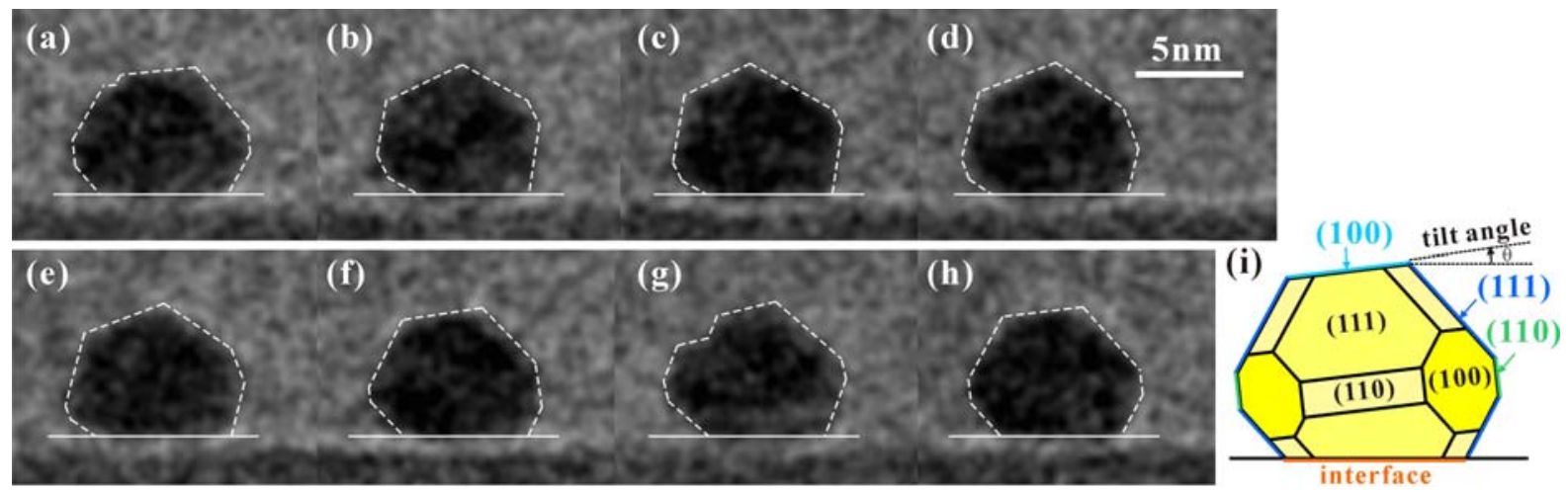

FIG. 1 (a)-(h) TEM images of an Au particle supported on $\mathrm{TiO}_{2}$, observing under reaction gas condition (1000 Pa; CO + Air), taken at intervals of 0.2 seconds. (i) Illustration of Wulff construction of (h).

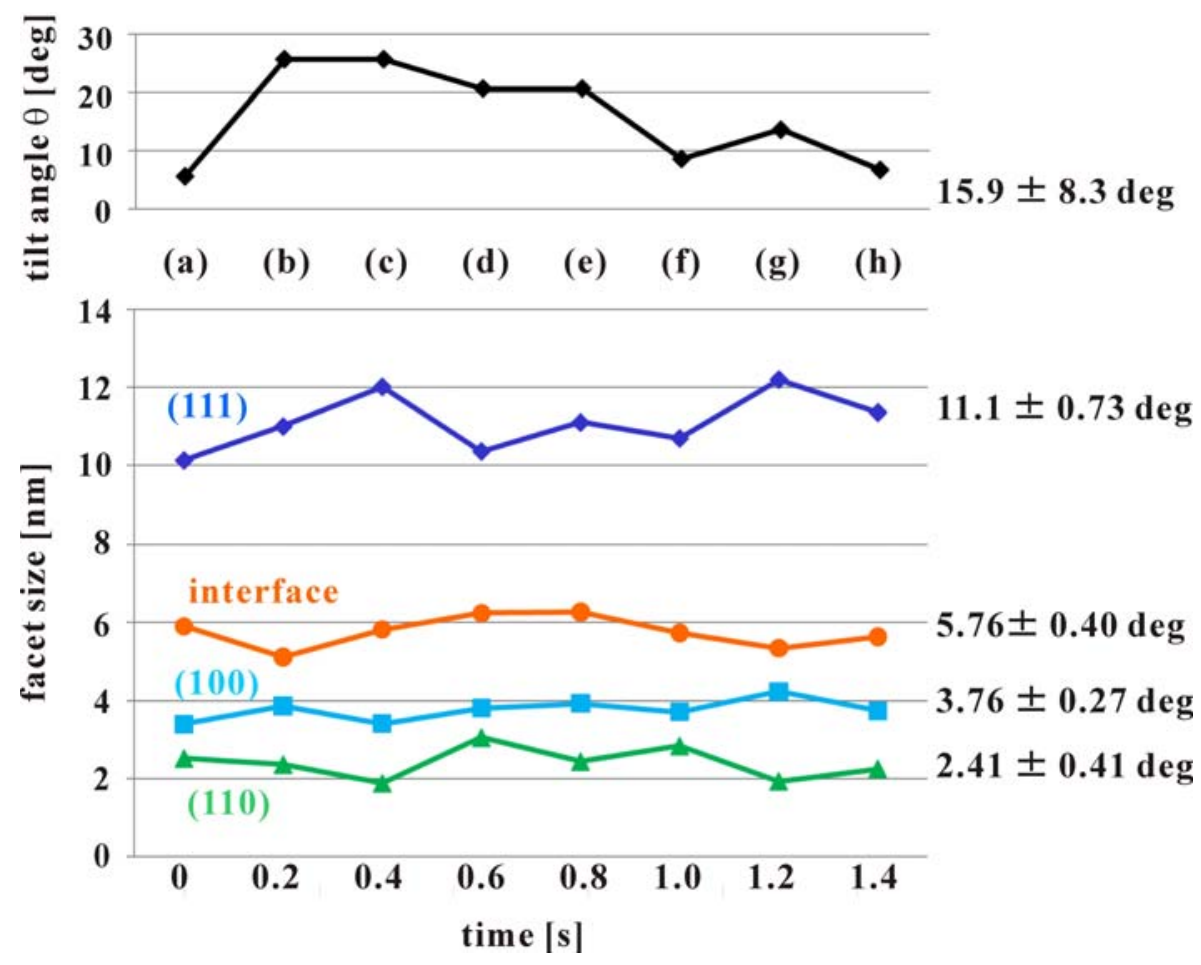

FIG. 2 Time-dependent changes of tilt angle of (100) facet (above) and sizes of three facets (below) of the Au particle measured in Fig. 1(a)-(h). 